\title{
Describing Variability of Inpatient Consultation Practices: Physician, Patient, and Admission Factors
}

\author{
Marika Kachman, $\mathrm{BA}^{1 *}$, Keme Carter, $\mathrm{MD}^{2}$, Vineet M Arora, MD, MAPP², Andrea Flores, $\mathrm{MA}^{2}$, \\ David O Meltzer, MD, PhD², Shannon K Martin, MD, MS²
}

${ }^{1}$ University of Chicago Pritzker School of Medicine, Chicago, Illinois; ${ }^{2}$ Department of Medicine, University of Chicago, Chicago, Illinois.

Appropriate use of consultation can improve patient outcomes, but inappropriate use may cause harm. Factors affecting the variability of inpatient consultation are poorly understood. We aimed to describe physician-, patient-, and admission-level factors influencing the variability of inpatient consultations on general medicine services. We conducted a retrospective study of patients hospitalized from 2011 to 2016 and enrolled in the University of Chicago Hospitalist Project, which included 6,153 admissions of 4,772 patients under 69 attendings. Consultation use varied widely; a 5.7-fold difference existed between the lowest (mean, 0.613) and highest (mean, 3.47) quartiles of use $(P<.01)$. In mixed-effect Poisson regression, consultations decreased over time, with $45 \%$ fewer consultations for admissions in 2015 than in 2011 $(P<.01)$. Patients on nonteaching hospitalist teams received $9 \%$ more consultations than did those on teaching services $(P=.02)$. Significant variability exists in inpatient consultation use. Further understanding may help to identify groups at high-risk for underuse/ overuse and aid in the development of interventions to improve high-value care. Journal of Hospital Medicine 2020;15:164-168. (c) 2020 Society of Hospital Medicine npatient consultation is an extremely common practice with the potential to improve patient outcomes significantly. ${ }^{1-3}$ However, variability in consultation practices may be risky for patients. In addition to underuse when the benefit is clear, the overuse of consultation may lead to additional testing and therapies, increased length of stay (LOS) and costs, conflicting recommendations, and opportunities for communication breakdown.

Consultation use is often at the discretion of individual providers. While this decision is frequently driven by patient needs, significant variation in consultation practices not fully explained by patient factors exists. ${ }^{1}$ Prior work has described hospital-level variation ${ }^{1}$ and that primary care physicians use more consultation than hospitalists. ${ }^{4}$ However, other factors affecting consultation remain unknown. We sought to explore physician-, patient-, and admission-level factors associated with consultation use on inpatient general medicine services.

\section{METHODS}

\section{Study Design}

We conducted a retrospective analysis of data from the University of Chicago Hospitalist Project (UCHP). UCHP is a longstand-

\footnotetext{
*Corresponding Author: Marika Kachman, BA; Email: mkachman@uchicago. edu; Telephone: 773-702-2604

Published online first February 19, 2020

Find Additional Supporting Information in the online version of this article.
}

Received: July 2, 2019; Revised: November 5, 2019;

Accepted: November 6, 2019

๑ 2020 Society of Hospital Medicine DOI 10.12788/jhm.3355 ing study of the care of hospitalized patients admitted to the University of Chicago general medicine services, involving both patient data collection and physician experience surveys. ${ }^{5}$ Data were obtained for enrolled UCHP patients between 2011-2016 from the Center for Research Informatics (CRI). The University of Chicago Institutional Review Board approved this study.

\section{Data Collection}

Attendings and patients consented to UCHP participation. Data collection details are described elsewhere., 5 Data from EpicCare (EpicSystems Corp, Wisconsin) and Centricity Billing (GE Healthcare, Illinois) were obtained via CRI for all encounters of enrolled UCHP patients during the study period $(N=218,591)$.

\section{Attending Attribution}

We determined attending attribution for admissions as follows: the attending author of the first history and physical (H\&P) was assigned. If this was unavailable, the attending author of the first progress note (PN) was assigned. For patients admitted by hospitalists on admitting shifts to nonteaching services (ie, service without residents/students), the author of the first PN was assigned if different from H\&P. Where available, attribution was corroborated with call schedules.

\section{Sample and Variables}

All encounters containing inpatient admissions to the University of Chicago from May 10, 2011 (Electronic Health Record activation date), through December 31, 2016, were considered for inclusion ( $N=51,171$, Appendix 1). Admissions including only documentation from ancillary services were excluded (eg, encounters for hemodialysis or physical therapy). Admissions 
TABLE 1. Attending, Patient, and Admission Demographics ${ }^{a}$

\begin{tabular}{|c|c|}
\hline \multicolumn{2}{|l|}{ Attending Physician Characteristics ( $N=69$ ) } \\
\hline Female, $n(\%)$ & $32(46.4 \%)$ \\
\hline Years in practice ${ }^{b}$ Mean (SD), median (IQR) & $11.9(8.4), 8(5-14)$ \\
\hline Specialty: General Internal Medicine, Medical Subspecialtyc & $61(88 \%), 8(12 \%)$ \\
\hline Hospitalist, n (\%) & $38(55.1 \%)$ \\
\hline \multicolumn{2}{|l|}{ Agreement with selected end of year survey statements ${ }^{d}, \mathrm{n}(\%)$} \\
\hline "I am satisfied with my ability to get to know patients." & $55(79.7 \%)$ \\
\hline "I am up to date on the practice of inpatient medicine." & $62(89.9 \%)$ \\
\hline "The interruption of my personal life by work is a problem." & $43(62.3 \%)$ \\
\hline \multicolumn{2}{|l|}{ Patient Characteristics $(\mathrm{N}=4,772)$} \\
\hline Female, n (\%) & $2,705(56.7 \%)$ \\
\hline Age - Mean (SD), median (IQR) & $61.0(19.5), 61(47-75)$ \\
\hline \multicolumn{2}{|l|}{ Admission Characteristics $(\mathrm{N}=6,153)$} \\
\hline LOS - Mean (SD), median (IQR) & $3.0(1.3), 3(2-4)$ \\
\hline Payor Status, n (\%): Medicare, Private Insurer, Medicaid, Uninsured & 2,406 (50.4\%), $968(20.3 \%), 1,195(25.0 \%), 87(2.4 \%)$ \\
\hline \multicolumn{2}{|l|}{ Hospitalist service admissions $(N=4,324)$} \\
\hline Hospitalist on teaching service, $\mathrm{n}(\%)$ & $2,769(64)$ \\
\hline Elixhauser Index: In-hospital Mortality - Mean (SD), median (IQR) & $5.1(8.4), 3(0-10)$ \\
\hline Elixhauser Index: 30-day readmission - Mean (SD), median (IQR) & $16.6(15.5), 14(2-27)$ \\
\hline Academic Tertile, n (\%): July - Oct, Nov - Feb, Jan - June & $2,477(40.3 \%), 1,962(31.9 \%), 1,714(27.9 \%)$ \\
\hline \multicolumn{2}{|c|}{ 'Because of missing data, numbers may not correspond to exact percentages. } \\
\hline \multicolumn{2}{|c|}{ bFrom medical school graduation to date of admission. } \\
\hline \multicolumn{2}{|l|}{ ‘General Medicine attendings who have subspecialty training. } \\
\hline \multicolumn{2}{|l|}{ ¿See Appendix 2 for all end of year survey questions. } \\
\hline Abbreviations: $I Q R$, interquartile range; $S D$, standard deviation. & \\
\hline
\end{tabular}

were limited to a length of stay $(\mathrm{LOS}) \leq 5$ days, corresponding to the average US inpatient LOS of 4.6 days, ${ }^{7}$ to minimize the likelihood of attending handoffs $(N=31,592)$. If attending attribution was not possible via the above-described methods, the admission was eliminated ( $N=3,103 ; 10.9 \%$ of admissions with LOS $\leq 5$ days). Finally, the sample was restricted to general medicine service admissions under attendings enrolled in UCHP who completed surveys. After the application of all criteria, 6,153 admissions remained for analysis.

The outcome variable was the number of consultations per admission, determined by counting the unique number of services creating clinical documentation, and subtracting one for the primary team. If the Medical/Surgical intensive care unit (ICU) was a service, then two were subtracted to account for the ICU transfer.

Attending years in practice (ie, years since medical school graduation) and gender were determined from public resources. Practice characteristics were determined from UCHP attending surveys, which address perceptions of workload and satisfaction (Appendix 2).

Patient characteristics (gender, age, Elixhauser Indices) and admission characteristics (LOS, season of admission, payor) were determined from UCHP and CRI data. The Elixhauser Index uses a well-validated system combining the presence/absence of 31 comorbidities to predict mortality and 30-day readmission. ${ }^{8}$ Elixhauser Indices were calculated using the "Creation of Elixhauser Comorbidity Index Scores 1.0" software. ${ }^{9}$ For admissions under hospitalist attendings, teaching/nonteaching team was ascertained via internal teaching service calendars.

\section{Analysis}

We used descriptive statistics to examine demographic characteristics. The difference between the lowest and highest quartile consultation use was determined via a two-sample $t$ test. Given the multilevel nature of our count data, we used a mixed-effects Poisson model accounting for within-group variation by clustering on attending and patient (3-level random-effects model). The analysis was done using Stata 15 (StataCorp, Texas).

\section{RESULTS}

From 2011 to 2016, 14,848 patients and 88 attendings were enrolled in UCHP; 4,772 patients (32\%) and 69 attendings (59.4\%) 
TABLE 2. Factors Associated with Consultation Variability by General Medicine Attendings ${ }^{\mathrm{a}, \mathrm{b}}$

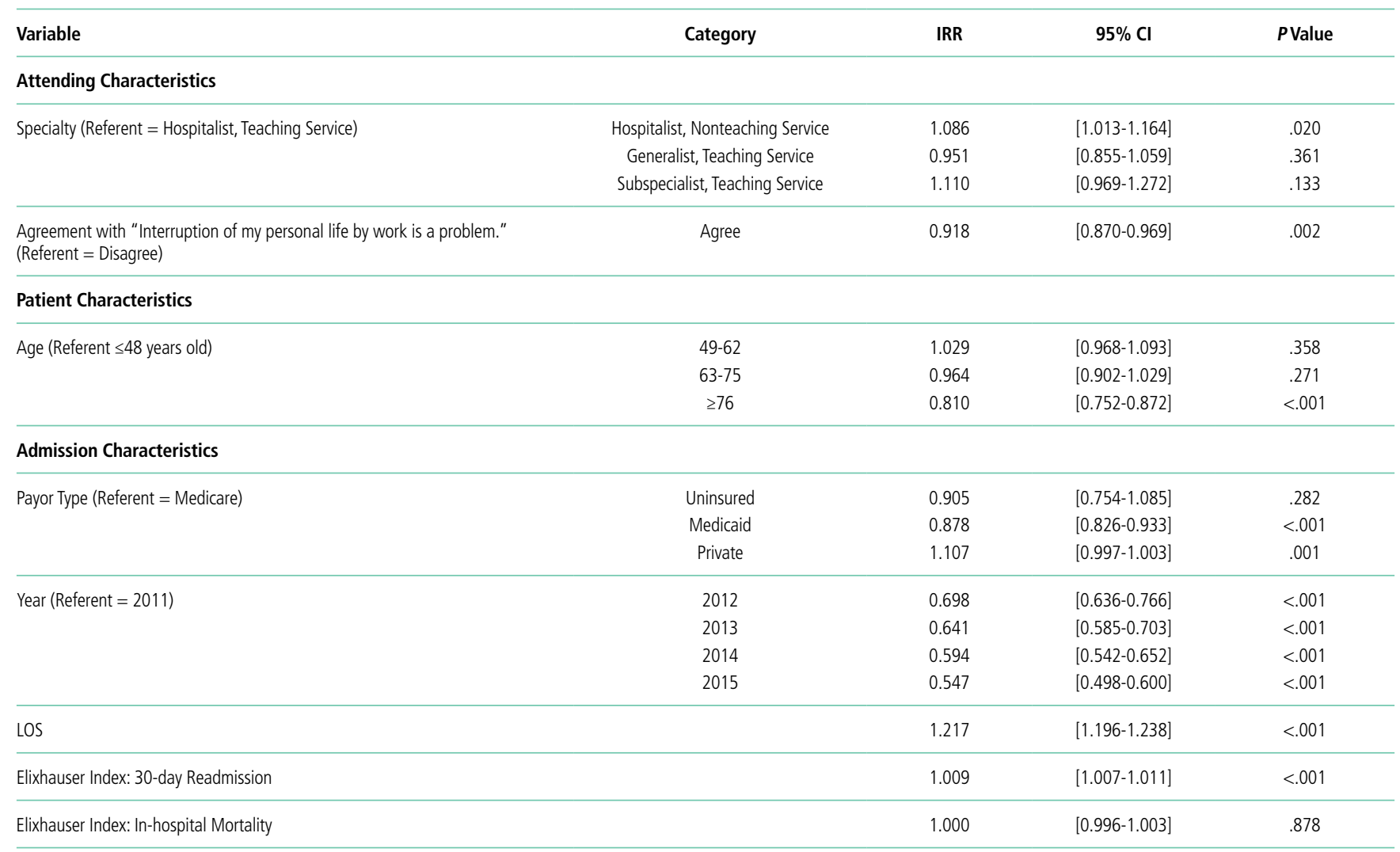

Multivariable mixed-effects Poisson regression model clustered on attending and patient, adjusted for additional physician-, patient- and admission-level factors. bee Appendix 3 for inclusion of full model.

Abbreviations: LOS, length of stay.

had data available and were included. Mean LOS was 3.0 days $(S D=1.3)$. Table 1 describes the characteristics of attendings, patients, and admissions.

Seventy-six percent of admissions included at least one consultation. Consultation use varied widely, ranging from 0 to 10 per admission (mean $=1.39$, median $=1$; standard deviation $[S D]=1.17$ ). The number of consultations per admission in the highest quartile of consultation frequency (mean $=3.47$, median $=3$ ) was 5.7 -fold that of the lowest quartile (mean $=0.613$, median $=1 ; P<.001$ ).

In multivariable regression, physician-, patient-, and admission-level characteristics were associated with the differential use of consultation (Table 2). On teaching services, consultations called by hospitalist vs nonhospitalist generalists did not differ $(P=.361)$. However, hospitalists on nonteaching services called $8.6 \%$ more consultations than hospitalists on teaching services $(P=.02)$. Attending agreement with survey item "The interruption of my personal life by work is a problem" was associated with $8.2 \%$ fewer consultations per admission $(P=.002)$.

Patients older than 75 years received 19\% fewer consultations compared with patients younger than 49 years $(P<.001)$. Compared with Medicare, Medicaid admissions had 12.2\% fewer consultations $(P<.001)$, whereas privately insured admissions had $10.7 \%$ more $(P=.001)$. The number of consultations per admission decreased every year, with $45.3 \%$ fewer consultations in 2015 than $2011(P<.001)$. Consultations increased by each $22 \%$ per day increase in $\operatorname{LOS}(P<.001)$.

\section{DISCUSSION}

Our analysis described several physician-, patient-, and admission-level characteristics associated with the use of inpatient consultation. Our results strengthen prior work demonstrating that patient-level factors alone are insufficient to explain consultation variability. ${ }^{1}$

Hospitalists on nonteaching services called more consultations, which may reflect a higher workload on these services. Busy hospitalists on nonteaching teams may lack time to delve deeply into clinical problems and require more consultations, especially for work with heavy cognitive loads such as diagnosis. "Outsourcing" tasks when workload increases occurs in other cognitive activities such as teaching. ${ }^{10}$ The association between work interrupting personal life and fewer consultations may also implicate the effects of time. Attendings who are experiencing work encroaching on their personal lives may be those spending more time with patients and consulting less. This finding merits further study, especially with increasing concern about balancing time spent in meaningful patient care activities with risk of physician burnout. 
This finding could also indicate that trainee participation modifies consultation use for hospitalists. Teaching service teams with more individual members may allow a greater pool of collective knowledge, decreasing the need for consultation to answer clinical questions. ${ }^{11}$ Interestingly, there was no difference in consultation use between generalists or subspecialists and hospitalists on teaching services, possibly suggesting a unique effect in hospitalists who vary clinical practice depending on team structure. These differences deserve further investigation, with implications for education and resource utilization.

We were surprised by the finding that consultations decreased each year, despite increasing patient complexity and availability of consultation services. This could be explained by a growing emphasis on shortening LOS in our institution, thus shifting consultative care to outpatient settings. Understanding these effects is critically important with growing evidence that consultation improves patient outcomes because these external pressures could lead to unintended consequences for quality or access to care.

Several findings related to patient factors additionally emerged, including age and insurance status. Although related to medical complexity, these effects persist despite adjustment, which raises the question of whether they contribute to the decision to seek consultation. Older patients received fewer consultations, which could reflect the use of more conservative practice models in the elderly, ${ }^{12}$ or ageism, which is associated with undertreatment. ${ }^{13}$ With respect to insurance status, Medicaid patients were associated with fewer consultations. This finding is consistent with previous work showing the decreased intensity of hospital services used for Medicaid patients. ${ }^{14}$

Our study has limitations. Our data were from one large urban academic center that limits generalizability. Although systematic and redundant, attending attribution may have been flawed: incomplete or erroneous documentation could have led to attribution error, and we cannot rule out the possibility of service handoffs. We used a LOS $\leq 5$ days to minimize this possibility, but this limits the applicability of our findings to longer admissions. Unsurprisingly, longer LOS correlated with the increased use of consultation even within our restricted sample, and future work should examine the effects of prolonged LOS. As a retrospective analysis, unmeasured confounders due to our limited adjustment will likely explain some findings, although we took steps to address this in our statistical design. Finally, we could not measure patient outcomes and, therefore, cannot determine the value of more or fewer consultations for specific patients or illnesses. Positive and negative outcomes of increased consultation are described, and understanding the impact of consultation is critical for further study.,3

\section{CONCLUSION}

We found that the use of consultation on general medicine services varies widely between admissions, with large differences between the highest and lowest frequencies of use. This variation can be partially explained by several physician-, patient-, and admission-level characteristics. Our work may help identify patient and attending groups at high risk for under- or overuse of consultation and guide the subsequent development of interventions to improve value in consultation. One additional consultation over the average LOS of 4.6 days adds $\$ 420$ per admission or $\$ 4.8$ billion to the 11.5 million annual Medicare admissions. ${ }^{15}$ Increasing research, guidelines, and education on the judicious use of inpatient consultation will be key in maximizing high-value care and improving patient outcomes.

\section{Acknowledgments}

The authors would like to acknowledge the invaluable support and assistance of the University of Chicago Hospitalist Project, the Pritzker School of Medicine Summer Research Program, the University of Chicago Center for Quality, and the University of Chicago Center for Health and the Social Sciences (CHeSS). The authors would additionally like to thank John Cursio, PhD, for his support and guidance in statistical analysis for this project.

Disclosures: The authors have nothing to disclose.

Funding: The authors acknowledge funding from the Alliance of Academic Internal Medicine 2017 Innovation Grant; the American Board of Medical Specialties Visiting Scholars Program; the National Heart, Lung, and Blood Institute Grant\# K24 - HL136859; and the National Institute on Aging Grant \#4T35AG029795-10. This project was also supported by the National Center for Advancing Translational Sciences of the National Institutes of Health (NIH) through Grant Number 5UL1TR002389-02 that funds the Institute for Translational Medicine.

Disclaimer: The content is solely the responsibility of the authors and does not necessarily represent the official views of the NIH. The funders had no role in the design of the study; the collection, analysis, and interpretation of the data; or the decision to approve publication of the finished manuscript. Preliminary results of this analysis were presented at the 2018 Society of Hospital Medicine Annual Meeting in Orlando, Florida. All coauthors have seen and agree with the contents of the manuscript. The submission is not under review by any other publication

\section{References}

1. Stevens JP, Nyweide D, Maresh S, et al. Variation in inpatient consultation among older adults in the United States. J Gen Intern Med. 2015;30(7):992999. https://doi.org/10.1007/s11606-015-3216-7.

2. Lahey T, Shah R, Gittzus J, Schwartzman J, Kirkland K. Infectious diseases consultation lowers mortality from Staphylococcus aureus bacteremia. Medicine (Baltimore). 2009;88(5):263-267. https://doi.org/10.1097/MD.0b013e3181b8f$\mathrm{ccb}$

3. Morrison RS, Dietrich J, Ladwig S, et al. Palliative care consultation teams cut hospital costs for Medicaid beneficiaries. Health Aff Proj Hope. 2011;30(3):454-463. https://doi.org/10.1377/hlthaff.2010.0929.

4. Stevens JP, Nyweide DJ, Maresh S, Hatfield LA, Howell MD, Landon BE. Comparison of hospital resource use and outcomes among hospitalists, primary care physicians, and other generalists. JAMA Intern Med. 2017;177(12):1781. https://doi.org/10.1001/jamainternmed.2017.5824.

5. Meltzer D. Effects of physician experience on costs and outcomes on an academic general medicine service: Results of a trial of hospitalists. Ann Intern Med. 2002;137(11):866. https://doi.org/10.7326/0003-4819-137-11200212030-00007.

6. Martin SK, Farnan JM, Flores A, Kurina LM, Meltzer DO, Arora VM. Exploring entrustment: Housestaff autonomy and patient readmission. Am J Med. 2014;127(8):791-797. https://doi.org/10.1016/j.amjmed.2014.04.013.

7. HCUP-US NIS Overview. https://www.hcup-us.ahrq.gov/nisoverview.jsp. Accessed July 7, 2017

8. Austin SR, Wong Y-N, Uzzo RG, Beck JR, Egleston BL. Why summary comorbidity measures such as the Charlson Comorbidity Index and Elixhauser Score work. Med Care. 2015;53(9):e65-e72. https://doi.org/10.1097/MLR. Ob013e318297429c.

9. Elixhauser Comorbidity Software. Elixhauser Comorbidity Software. https://www.hcup-us.ahrq.gov/toolssoftware/comorbidity/comorbidity. jsp\#references. Accessed May 13, 2019.

10. Roshetsky LM, Coltri A, Flores A, et al. No time for teaching? Inpatient at- 
tending physicians' workload and teaching before and after the implementation of the 2003 duty hours regulations. Acad Med J Assoc Am Med Coll. 2013;88(9):1293-1298. https://doi.org/10.1097/ACM.0b013e31829eb795.

11. Barnett ML, Boddupalli D, Nundy S, Bates DW. Comparative accuracy of diagnosis by collective intelligence of multiple physicians vs individual physicians. JAMA Netw Open. 2019;2(3):e190096. https://doi.org/10.1001/jamanetworkopen.2019.0096.

12. Aoyama T, Kunisawa S, Fushimi K, Sawa T, Imanaka Y. Comparison of surgical and conservative treatment outcomes for type $A$ aortic dissection in elderly patients. J Cardiothorac Surg. 2018;13(1):129. https://doi.org/10.1186/ s13019-018-0814-6.
13. Lindau ST, Schumm LP, Laumann EO, Levinson W, O'Muircheartaigh CA, Waite LJ. A study of sexuality and health among older adults in the United States. N Engl J Med. 2007;357(8):762-774. https://doi.org/10.1056/NEJMoa067423.

14. Yergan J, Flood AB, Diehr P, LoGerfo JP. Relationship between patient source of payment and the intensity of hospital services. Med Care. 1988;26(11):1111-1114. https://doi.org/10.1097/00005650-198811000-00009.

15. Center for Medicare and Medicaid Services. MDCR INPT HOSP 1.; 2008. https://www.cms.gov/Research-Statistics-Data-and-Systems/Statistics-Trends-and-Reports/CMSProgramStatistics/2013/Downloads/ MDCR_UTIL/CPS_MDCR_INPT_HOSP_1.pdf. Accessed April 15, 2018. 